\title{
Radiation Dose of the P-Glycoprotein Tracer ${ }^{11} \mathrm{C}$-Laniquidar
}

\author{
Andrey Postnov ${ }^{1}$, Femke E. Froklage ${ }^{2,3}$, Arthur van Lingen ${ }^{1}$, Jaap C. Reijneveld ${ }^{2}$, N. Harry Hendrikse ${ }^{1,4}$, \\ Albert D. Windhorst ${ }^{1}$, Robert C. Schuit ${ }^{1}$, Jonas Eriksson ${ }^{1}$, Adriaan A. Lammertsma ${ }^{1}$, and Marc C. Huisman ${ }^{1}$ \\ ${ }^{I}$ Departments of Radiology and Nuclear Medicine, VU University Medical Center, Amsterdam, The Netherlands; ${ }^{2}$ Department of \\ Neurology, VU University Medical Center, Amsterdam, The Netherlands; ${ }^{3}$ Epilepsy Institute in The Netherlands Foundation (SEIN), \\ Heemstede, The Netherlands; and ${ }^{4}$ Clinical Pharmacology and Pharmacy, VU University Medical Center, Amsterdam, The Netherlands
}

Resistance to current drug therapy is an important issue in the treatment of epilepsy. Inadequate access of central nervous system drugs to their targets in the brain may be caused by overexpression or overactivity of multidrug transporters, such as P-glycoprotein (P-gp), at the blood-brain barrier. Laniquidar, an inhibitor of P-gp, has been labeled with ${ }^{11} \mathrm{C}$ for use in PET studies of P-gp expression in humans. Given potential interspecies differences in biodistribution, the purpose of this study was to ensure safe use of ${ }^{11} \mathrm{C}$-laniquidar by determining the dosimetry of ${ }^{11} \mathrm{C}$-laniquidar using whole-body PET studies. Methods: Six healthy volunteers were subjected to a series of 10 whole-body PET scans within approximately $70 \mathrm{~min}$. Five blood samples were taken during the series. Results: High uptake of ${ }^{11} \mathrm{C}$ laniquidar was seen in liver, spleen, kidneys, and lung, whereas brain uptake was low. The effective dose for ${ }^{11} \mathrm{C}$-laniquidar was $4.76 \pm$ 0.13 and $3.69 \pm 0.01 \mu \mathrm{Sv} \cdot \mathrm{MBq}^{-1}$ for women and men, respectively. Conclusion: Biodistribution and measured effective dose indicate that ${ }^{11} \mathrm{C}$-laniquidar is a safe tracer for PET imaging, with a total dose of about $2 \mathrm{mSv}$ for a brain PET/CT protocol.

Key Words: P-glycoprotein; PET; ${ }^{11} \mathrm{C}$-laniquidar; dosimetry; multidrug transporter

J Nucl Med 2013; 54:2101-2103

DOI: 10.2967/jnumed.113.120857

$\mathbf{R}$ esistance to drug therapy affects approximately $30 \%$ of all patients with epilepsy (1) and may be due, at least in part, to decreased passage of antiepileptic drugs across the blood-brain barrier. Uptake and efflux drug transporters play a major functional role in regulating drug entry into the brain. Two large and important drug transporter families are the organic anion-transporting polypeptide (OATP) family and the adenosine triphosphate-binding cassette transporter superfamily $(2,3)$. Several members of both families are expressed at the human blood-brain barrier, including OATP1A2, OATP1C1, and OATP3A1 (members of the OATP family) and P-glycoprotein (P-gp), breast cancer resistance protein, and multidrug resistance protein 4 (members of the adenosine triphosphate-binding transporter superfamily) $(2,3)$. In this paper, the focus is on the most widely studied efflux transporter, P-gp. It has

Received Jan. 31, 2013; revision accepted Aug. 16, 2013.

For correspondence or reprints contact: Femke E. Froklage, Department of Neurology, ZH-2F19, VU University Medical Center, P.O. Box 7057, 1007 MB Amsterdam, The Netherlands.

E-mail: f.froklage@vumc.nl

Published online Oct. 3, 2013.

COPYRIGHT (C 2013 by the Society of Nuclear Medicine and Molecular Imaging, Inc. been proposed that changes in P-gp expression or function at the blood-brain barrier play an important role in pharmacoresistance in epilepsy (4). The multidrug transporter P-gp and other efflux transporters actively transport substrates, including many central nervous system drugs, against a concentration gradient from brain to blood and cerebrospinal fluid. Hence, overexpression or increased activity of the transporter system may result in reduced tissue concentrations of central nervous system drugs in the brain, thereby greatly limiting their therapeutic efficacy. There are two case reports suggesting that inhibiting P-gp in medically refractory epilepsy patients decreases seizure frequency, at least temporarily $(5,6)$. P-gp functionality can be assessed in vivo by means of $(R)-{ }^{11} \mathrm{C}$-verapamil and ${ }^{11} \mathrm{C}-\mathrm{N}$-desmethylloperamide PET scans $(7,8)$. However, at present overexpression of $\mathrm{P}$-gp in refractory patients can be confirmed only by examining postmortem brain tissue or surgically removed brain tissue. Therefore, the availability of noninvasive imaging techniques that would allow assessment of the distribution of P-gp in the brain is of vital importance. Laniquidar is an inhibitor of P-gp (9) and therefore should bind proportionally to P-gp density. Recently, this compound was labeled with ${ }^{11} \mathrm{C}$ and its biodistribution in rats was evaluated (10). Previously, however, metabolic profiles for the P-gp substrate tracer $(R)-{ }^{11} \mathrm{C}$-verapamil were shown to differ substantially between species $(11,12)$, as may also be the case for ${ }^{11} \mathrm{C}$-laniquidar. Therefore, the purpose of the present study was to determine the radiation dosimetry of ${ }^{11} \mathrm{C}$-laniquidar based on biodistribution studies in humans.

\section{MATERIALS AND METHODS}

\section{Subjects}

Six healthy subjects with no evidence of renal or hepatic dysfunction (4 men and 2 women) were included. They had a mean age $( \pm \mathrm{SD})$ of $43 \pm 18 \mathrm{y}$ and a mean body weight of $86 \pm 23 \mathrm{~kg}$. Before inclusion, each participant signed a protocol-specific informed consent form. The study was approved by the Medical Ethics Review Committee of the VU University Medical Center.

\section{Synthesis of ${ }^{11} \mathrm{C}$-Laniquidar}

${ }^{11} \mathrm{C}$-laniquidar was synthesized according to good-manufacturingpractice standards as described previously (10), with some modifications to enable human use. Briefly, $2.1 \pm 0.6 \mathrm{GBq}$ of ${ }^{11} \mathrm{C}-$ laniquidar were obtained via alkylation of $O$-desmethyl laniquidar (R102207) with ${ }^{11} \mathrm{C}$-methyl triflate and, after purification by highperformance liquid chromatography, reformulated in a sterile aqueous solution of $0.9 \% \mathrm{NaCl}, 2.5 \%$ polysorbate 80 , and $8.5 \%$ ethanol. Radiochemical purity was higher than $98 \%$, and no chemical impurities were observed as assessed by radio/ultraviolet high-performance liquid chromatography. The identity of the product was confirmed by comparing its retention time with that of authentic laniquidar. The mean specific activity at the time of injection was $70 \pm 24 \mathrm{GBq} \cdot \mu \mathrm{mol}^{-1}$. 


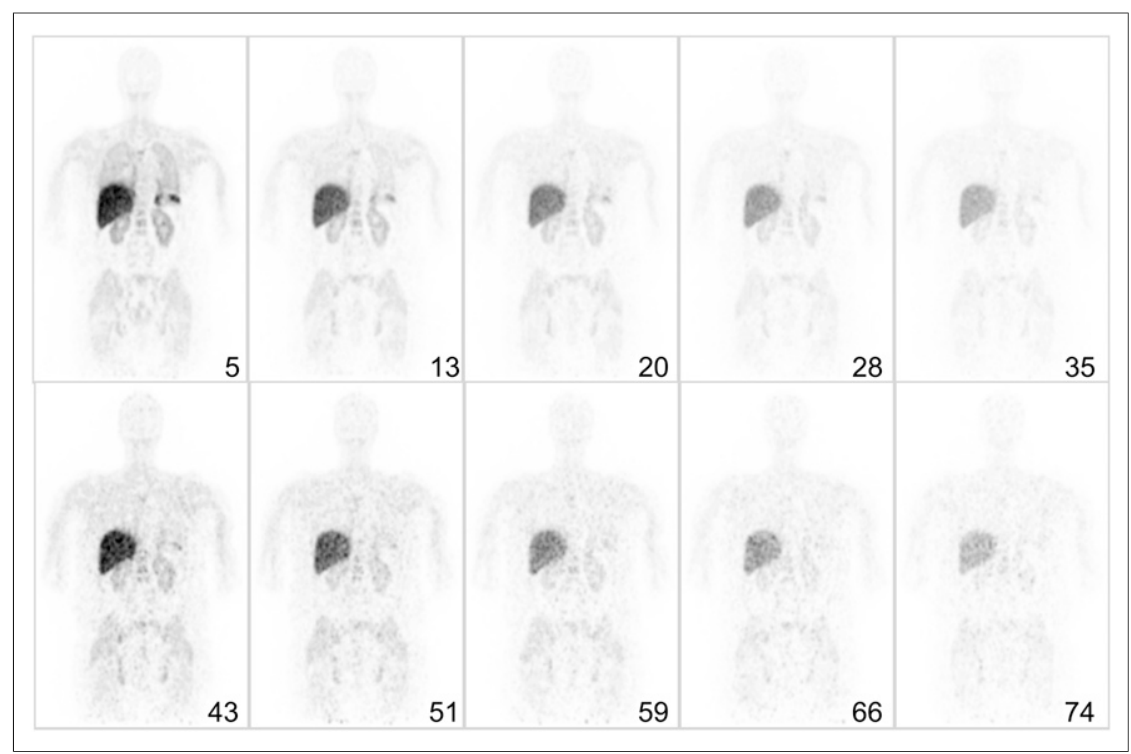

FIGURE 1. Coronal views showing biodistribution of ${ }^{11} \mathrm{C}$-laniquidar as function of time (numbers in panels indicate start time of scan after injection [min]) for subject F-1. No decay correction was performed between images; that is, images illustrate sum of biologic clearance and physical decay. For upper row, image scale runs between SUV of 0 (white) and 16 (black). For lower row, these numbers are 0 and 4 , respectively.

\section{Scan Protocol}

Each subject received 2 venous cannulas. One cannula was used for tracer administration and the other for blood sampling (the use of two separate cannulas excludes the possibility of contamination of blood samples with radioactivity traces left from the intravenous administration). Subsequently, the subjects were positioned on the bed of a Gemini TF 64 PET/CT scanner (Philips). The mean and SD of the administered mass of ${ }^{11} \mathrm{C}$-laniquidar were $3.5 \pm 1.4 \mu \mathrm{g}$ (range, $2.2-$ $5.9 \mu \mathrm{g}$ ). The mean administered activity was $347 \pm 63 \mathrm{MBq}$ (range, 222-396 MBq). After a 35-mAs low-dose whole-body (WB) CT scan, a series of $10 \mathrm{WB}$ sweeps was started (30 s per bed position, typically 11 bed positions), covering the interval between 0 and about $70 \mathrm{~min}$ after tracer injection. Five blood samples per subject were taken manually, one after each odd-numbered WB scan.

\section{Data Analysis}

Radioactivity in $0.5-\mathrm{mL}$ blood samples was measured with a $\gamma$ counter (Perkin Elmer). All WB PET scans were reconstructed using the standard WB time-of-flight reconstruction algorithm, including normalization of the data as well as scatter, attenuation, decay, and dead-time corrections (13). In particular, decay correction was performed within each scan (to account for the time difference between the various bed positions) but not for each scan with respect to the time of injection (since residence time calculations should be performed on non-decay-corrected source organ time-activity data). Regions of interest were defined for organs that showed positive image contrast on a PET image: myocardium, liver, kidneys, spleen, and lungs. With the exception of the lungs, these regions were delineated on an early PET scan. Organs that did not have a positive contrast in the PET scan were regarded as a part of the remainder of the body in the subsequent calculation of the radiation-absorbed dose. In addition, the brain was delineated on the CT scan, because brain imaging is the intended use of the tracer. Both CT regions were automatically segmented on the basis of a Hounsfield unit threshold. The lung region of interest was manually edited if the location on the respiration-averaged PET scan differed from that on the CT scan. All regions of interest were copied onto all PET scans to generate mean time-activity concentration curves per organ. At the end of the PET studies, activity remained in various organs. To account for this residual activity, each individual time-activity curve was extrapolated to infinity by assuming only physical decay from the end of the last WB scan onward. In this extrapolation, biologic clearance is ignored, resulting in a worst-case estimate of residual activity and thus an upper limit of the estimated radiation dose. The red marrow activity concentration was assumed to be one third of the whole-blood activity concentration (14). The area under each time-activity curve was integrated, and the residence time for each organ was obtained through multiplication of this area by the scaled organ mass from reference man or woman and division by the injected dose. To calculate the residence time for the remainder of the body, residence times of all source organs were summed and subtracted from the fixed theoretic value in the absence of excretion. The software package OLINDA was used to calculate the effective dose in $\mu \mathrm{Sv} \cdot \mathrm{MBq}^{-1}$ according to International Commission on Radiological Protection publication 60 (15) tissue weighting factors (16).

\section{RESULTS}

There were no adverse or clinically detectable pharmacologic effects in any of the 6 subjects. No significant changes in vital signs were observed. No patient motion between CT and PET scans was observed. A typical coronal WB slice of ${ }^{11} \mathrm{C}$-laniquidar uptake as a function of time is shown in Figure 1. Urinary bladder and testes did not show significant tracer accumulation and therefore were not included in dose calculations. Figure 2 shows timeactivity curves for individual organs and for blood sampling averaged over all subjects. Organ, red marrow, and effective doses are given in Table 1. Data for individual subjects are presented to illustrate interindividual variability. An average effective dose of

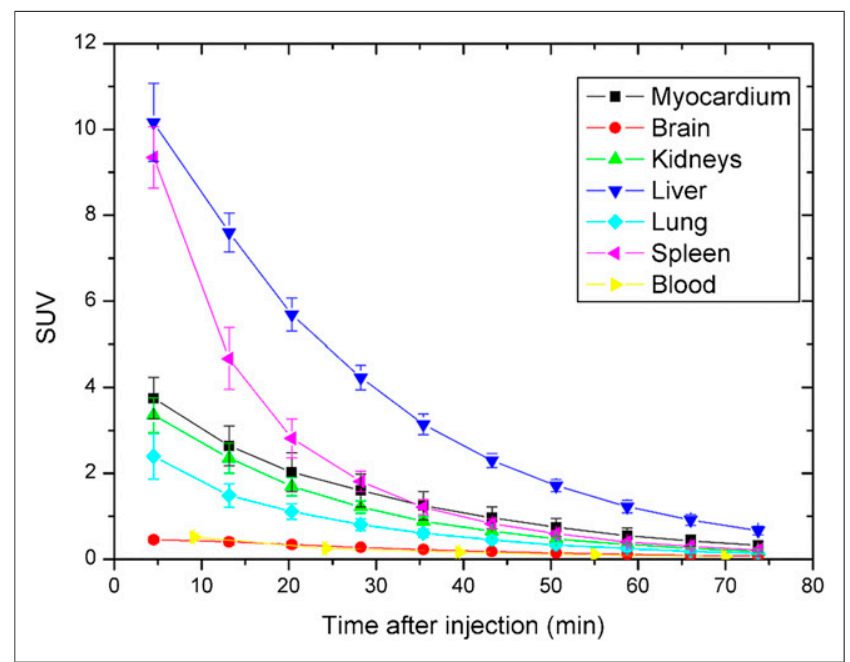

FIGURE 2. Time-activity curves of individual organs and whole blood averaged over all patients. 
TABLE 1

Absorbed Organ Doses and Effective Dose for Individual Subjects ( $\mu$ Sv. $\mathrm{MBq}^{-1}$ )

\begin{tabular}{|c|c|c|c|c|c|c|c|c|c|c|}
\hline Subject no. & Kidneys & Liver & Lungs & Spleen & Myocardium & Brain & $\mathrm{RM}$ & Gonads & RoB & $E$ \\
\hline $\mathrm{F}-1$ & 9.35 & 3.29E1 & 7.55 & 1.68 E1 & 1.30 E1 & 2.09 & 2.63 & 2.87 & 3.70 & 4.90 \\
\hline F-2 & 9.06 & 2.76 E1 & 6.14 & $1.59 \mathrm{E} 1$ & 9.81 & 1.79 & 2.69 & 3.12 & 3.68 & 4.59 \\
\hline$F-3$ & 8.97 & $2.70 \mathrm{E} 1$ & 8.28 & 1.82 E1 & 1.43 E1 & 2.48 & 2.73 & 3.02 & 3.68 & 4.80 \\
\hline $\mathrm{F}-4$ & 9.65 & 3.09 E1 & 6.58 & $1.43 \mathrm{E} 1$ & 1.04 E1 & 2.04 & 2.69 & 3.00 & 3.69 & 4.74 \\
\hline F-avg & 9.26 & 2.96 E1 & 7.14 & $1.63 \mathrm{E} 1$ & 1.19 E1 & 2.10 & 2.69 & 3.00 & 3.69 & 4.76 \\
\hline F-SD & 0.31 & 2.79 & 0.96 & 1.64 & 2.13 & 0.29 & 0.04 & 0.10 & 0.01 & 0.13 \\
\hline$M-1$ & 8.45 & 2.31 E1 & 4.71 & $1.29 \mathrm{E} 1$ & 8.57 & 1.93 & 2.23 & 1.92 & 2.93 & 3.68 \\
\hline M-2 & 8.22 & 2.24 E1 & 5.06 & 1.31 E1 & 7.73 & 2.00 & 2.26 & 1.94 & 2.93 & 3.70 \\
\hline M-avg & 8.34 & 2.28 E1 & 4.89 & 1.30 E1 & 8.15 & 1.97 & 2.25 & 1.93 & 2.93 & 3.69 \\
\hline M-SD & 0.16 & 0.49 & 0.25 & 0.14 & 0.59 & 0.05 & 0.02 & 0.01 & - & 0.01 \\
\hline
\end{tabular}

$\mathrm{RM}$ = red marrow; RoB = remainder of body; $\mathrm{E}=$ effective dose according to International Commission on Radiological Protection publication 60 (15); avg = average.

$4.76 \pm 0.13$ and $3.69 \pm 0.01 \mu \mathrm{Sv} \cdot \mathrm{MBq}^{-1}$ was obtained for women and men, respectively.

\section{DISCUSSION}

The measurement protocol allowed for good visualization of tracer kinetics in the body. There was no indication of patient motion either between the CT and the PET scans or during the PET scans. The biodistribution of ${ }^{11} \mathrm{C}$-laniquidar showed highest uptake in liver, followed by spleen, kidneys, and lung. In rats, highest uptake was seen in lung, followed by liver, spleen, and kidney (10). It is not known why lung uptake seems to be species-dependent. All time-activity curves were descending, allowing for a reasonable estimate of the residence time for the interval after the scanning period. The absence of contrast in the lumbar vertebrae at the end of the scanning period suggests no active uptake in the bone marrow, allowing for an estimate of the bone marrow residence time from the blood samples. Organ and effective doses reproduced well despite the small number of female $(n=4)$ and male $(n=2)$ subjects. The measured average effective dose of $4.40 \pm 0.56 \mu \mathrm{Sv} \cdot \mathrm{MBq}^{-1}$ is within the range observed for other ${ }^{11} \mathrm{C}$-based tracers (17). The liver receives the highest organ dose of approximately $25 \mu \mathrm{Sv} \cdot \mathrm{MBq}^{-1}$. A typical injection of $370 \mathrm{MBq}$ would lead to a total dose of approximately $2 \mathrm{mSv}$ for a brain PET/CT protocol (including a low-dose CT scan).

\section{CONCLUSION}

In this dosimetry study, the average effective dose derived from ${ }^{11} \mathrm{C}$-laniquidar was $4.40 \pm 0.56 \mu \mathrm{Sv} \cdot \mathrm{MBq}^{-1}$. Therefore, ${ }^{11} \mathrm{C}$-laniquidar is safe for PET imaging of P-gp expression on the current generation of PET/CT scanners.

\section{DISCLOSURE}

The costs of publication of this article were defrayed in part by the payment of page charges. Therefore, and solely to indicate this fact, this article is hereby marked "advertisement" in accordance with 18 USC section 1734. This work was supported by the European Community's Seventh Framework Programme (FP7/2007-2013) under grant agreement no. 201380. No other potential conflict of interest relevant to this article was reported.

\section{ACKNOWLEDGMENTS}

We thank Prof. Ronald Boellaard for fruitful discussions and Dr. Marieke J. Hazewinkel for help with determining the regions of interest.

\section{REFERENCES}

1. Kwan P, Brodie MJ. Early identification of refractory epilepsy. $N$ Engl J Med. 2000;342:314-319.

2. Froklage FE, Reijneveld JC, Heimans JJ. Central neurotoxicity in cancer chemotherapy: pharmacogenetic insights. Pharmacogenomics. 2011;12:379-395.

3. Kalliokoski A, Niemi M. Impact of OATP transporters on pharmacokinetics. Br J Pharmacol. 2009; 158:693-705.

4. Löscher W. Animal models of drug-resistant epilepsy. Novartis Found Symp. 2002;243:149-159.

5. Iannetti P, Spalice A, Parisi P. Calcium-channel blocker verapamil administration in prolonged and refractory status epilepticus. Epilepsia. 2005;46:967-969.

6. Summers MA, Moore JL, McAuley JW. Use of verapamil as a potential Pglycoprotein inhibitor in a patient with refractory epilepsy. Ann Pharmacother. 2004;38:1631-1634.

7. Feldmann M, Koepp M. P-glycoprotein imaging in temporal lobe epilepsy: in vivo PET experiments with the Pgp substrate $\left[{ }^{11} \mathrm{C}\right]$-verapamil. Epilepsia. 2012;53(suppl 6):60-63.

8. Kreisl WC, Liow JS, Kimura N, et al. P-glycoprotein function at the blood-brain barrier in humans can be quantified with the substrate radiotracer ${ }^{11} \mathrm{C}-\mathrm{N}$-desmethylloperamide. J Nucl Med. 2010;51:559-566.

9. van Zuylen L, Sparreboom A, van der Gaast A, et al. The orally administered Pglycoprotein inhibitor R101933 does not alter the plasma pharmacokinetics of docetaxel. Clin Cancer Res. 2000;6:1365-1371.

10. Luurtsema G, Schuit RC, Klok RP, et al. Evaluation of $\left[{ }^{11} \mathrm{C}\right]$ laniquidar as a tracer of P-glycoprotein: radiosynthesis and biodistribution in rats. Nucl Med Biol. 2009;36:643-649.

11. Lubberink M, Luurtsema G, van Berckel BN, et al. Evaluation of tracer kinetic models for quantification of P-glycoprotein function using $(\mathrm{R})-\left[{ }^{11} \mathrm{C}\right]$ verapamil and PET. J Cereb Blood Flow Metab. 2007;27:424-433.

12. Luurtsema G, Molthoff CF, Schuit RC, Windhorst AD, Lammertsma AA, Franssen EJ. Evaluation of (R)-[ $\left.{ }^{11} \mathrm{C}\right]$ verapamil as PET tracer of P-glycoprotein function in the blood-brain barrier: kinetics and metabolism in the rat. Nucl Med Biol. 2005;32:87-93.

13. Surti S, Kuhn A, Werner ME, Perkins AE, Kolthammer J, Karp JS. Performance of Philips Gemini TF PET/CT scanner with special consideration for its time-offlight imaging capabilities. J Nucl Med. 2007;48:471-480.

14. Hindorf C, Glatting G, Chiesa C, Linden O, Flux G. EANM Dosimetry Committee guidelines for bone marrow and whole-body dosimetry. Eur J Nucl Med Mol Imaging. 2010;37:1238-1250.

15. 1990 Recommendations of the International Commission on Radiological Protection: ICRP publication 60. Ann ICRP. 1991;21(1-3).

16. Stabin MG, Sparks RB, Crowe E. OLINDA/EXM: the second-generation personal computer software for internal dose assessment in nuclear medicine. $\mathrm{J} \mathrm{Nucl}$ Med. 2005;46:1023-1027.

17. van der Aart J, Hallett WA, Rabiner EA, Passchier J, Comley RA. Radiation dose estimates for carbon-11-labelled PET tracers. Nucl Med Biol. 2012;39:305-314. 